\title{
Direct Probing of Quantum Phase Space by Photon Counting
}

\author{
Konrad Banaszek and Krzysztof Wódkiewicz* \\ Instytut Fizyki Teoretycznej, Uniwersytet Warszawski, 00-681 Warszawa, Hoża 69, Poland
}

(February 29, 1996)

We propose a very simple experimental setup to measure, via photon counting, the overlap of the Wigner functions characterizing two single mode light beams. We show that this scheme can be applied to determine directly the phase space quasiprobability distribution of the single mode field and in a certain limit the Wigner function can be measured without use of tomographic reconstruction algorithms. The deleterious effects of non-unit photodetector efficiency are analyzed.

PACS number(s): 42.50.Dv, 03.65.Bz

The concept of phase space, fundamental in classical mechanics, remains useful when passing to quantum theory. It is well known that quantum states can be fully characterized by the Wigner function defined in the phase space and that the quantum expectation values can be represented as statistical averages of the phase space variables [1]. In quantum optics this formalism provides a convenient framework for discussion of many topics [2].

An exciting problem explored recently is the experimental determination of the Wigner function of a single light mode. It was first shown theoretically by Vogel and Risken [3] that quadrature amplitude distributions measured in homodyne detection provide enough data to perform a complete reconstruction of the Wigner function. This method, called quantum tomography, was successfully realized in a series of experiments 沺. The quantity recorded in the quantum tomography experiments was the statistics of the count difference of photodetectors facing the signal field superimposed on a strong coherent field. The Wigner function was reconstructed from these data using numerical algorithms of the inverse Radon transform. There are also other known methods for the complete experimental characterization of the single mode state: heterodyne [5] and double homodyne [6 8] detection, where the so-called $Q$ function of the signal field, which is a smoothed Wigner function, is measured.

In this Letter we propose an alternative scheme for the phase space measurement. Given two single mode light beams we present an extremely simple experimental setup to measure the overlap of the Wigner functions characterizing these field. Moreover we show that the Wigner functions can be relatively rescaled by an arbitrary positive factor. The measurement is performed by optical means and only a trivial arithmetic operation has to be done on the data recorded from the photodetector.

Before we discuss the proposed measurement, we review briefly, using a Heisenberg-picture-type of approach, the properties of the phase space distributions used in this Letter. The Wigner function or the $Q$ function are examples of more general $s$-parameterized quasiprobability distributions $W(\alpha ; s)$ in the complex $\alpha$ phase space [9], with $s=0$ corresponding to the Wigner function $W(\alpha)=W(\alpha ; 0)$. These distributions can be written as expectation values of the following normally ordered operator:

$$
\hat{U}(\alpha ; s)=\frac{2}{\pi(1-s)}: \exp \left(-\frac{2}{1-s}\left(\alpha^{*}-\hat{a}^{\dagger}\right)(\alpha-\hat{a})\right):
$$

where $\hat{a}^{\dagger}$ and $\hat{a}$ are the single mode photon creation and annihilation operators. In the proposed measurement scheme we utilize the simple fact that the single mode Wigner function at the origin of the complex phase space $W(0)$ can be directly computed from the distribution of counts measured by a photodetector facing this mode. Indeed for $\alpha=0$ and $s=0$ the operator (11) can be written in the following equivalent forms:

$$
\begin{aligned}
\hat{U}(0 ; 0) & =\frac{2}{\pi}: \exp \left(-2 \hat{a}^{\dagger} \hat{a}\right): \\
& =\frac{2}{\pi} \sum_{n=0}^{\infty}(-1)^{n}: e^{-\hat{a}^{\dagger} \hat{a}} \frac{\left(\hat{a}^{\dagger} \hat{a}\right)^{n}}{n !}: \\
& =\frac{2}{\pi} \sum_{n=0}^{\infty}(-1)^{n}|n\rangle\langle n|
\end{aligned}
$$

where we have used the normally ordered operator representation of the $n$ photon number projection operator. The quantum expectation value of this operator gives:

$$
W(0)=\langle\hat{U}(0 ; 0)\rangle=\frac{2}{\pi} \sum_{n=0}^{\infty}(-1)^{n} p_{n}
$$

where the value $p_{n}$ appearing in this expansion is just the probability of counting $n$ photons by an ideal photodetector. Thus the photoncount statistics allows to calculate the Wigner function at the origin of the phase space. It has been pointed out by Royer [10] that the ability to measure $W(0)$ allows us to scan the complete Wigner function by shifting the system or equivalently the frame of reference in the phase space. Although in principle this shifting of the reference frame might be realized in quantum optics, its experimental realization would encounter difficulties. Therefore we will utilize the results of the measurement of $W(0)$ in an alternative way, providing a much more feasible experimental scheme. The 
proposed scheme is very general and we believe that it may find many various applications and generalizations. In the present Letter we will discuss in detail one specific example motivated by the quantum tomography experiments.

The proposed setup is presented in Fig. 1. We will take the detected field to be a superposition of two single mode fields, which we will call the signal and the probe, and denote their annihilation operators by $\hat{a}_{S}$ and $\hat{a}_{P}$ respectively. Such a combination can be easily realized by means of a beamsplitter. As it is well known [2], the action of the beamsplitter is described by an $\mathrm{SU}(2)$ transformation between the annihilation operators of the incoming and outgoing modes. Since the phase shifts appearing in this transformation can be eliminated by an appropriate redefinition of the modes, the annihilation operator of the outgoing mode falling onto the detector face $\hat{a}_{\text {out }}$ can be assumed to be a combination

$$
\hat{a}_{\text {out }}=\sqrt{T} \hat{a}_{S}-\sqrt{1-T} \hat{a}_{P}
$$

where $T$ is the beamsplitter power transmissivity The Wigner function of the outgoing mode at the phase space origin is given in terms of the incoming modes by the expectation value of

$$
\begin{aligned}
\hat{U}_{\text {out }}(0 ; 0)= & \frac{2}{\pi}: \exp \left(-2 \hat{a}_{\text {out }}^{\dagger} \hat{a}_{\text {out }}\right): \\
= & \frac{2}{\pi}: \exp \left(-2 T\left(\hat{a}_{S}^{\dagger}-\sqrt{(1-T) / T} \hat{a}_{P}^{\dagger}\right)\right. \\
& \left.\times\left(\hat{a}_{S}-\sqrt{(1-T) / T} \hat{a}_{P}\right)\right): .
\end{aligned}
$$

This simple relation provides an interesting link between the detected quantity and the $S$ mode. Let us for example consider a simple case when the probe field is a coherent state $\hat{a}_{P}|\alpha\rangle=\alpha|\alpha\rangle$ uncorrelated with the signal mode. Performing the quantum average over the $P$ mode in Eq. (5) is straightforward due to the normal ordering of the operators. Taking the expectation value over the signal and using the definition (1) we obtain that the Wigner function (3) for the outgoing mode is proportional to an $s=1-1 / T$ ordered quasidistribution function of the $S$ mode:

$$
W_{\text {out }}(0)=\frac{1}{T} W_{S}\left(\sqrt{\frac{1-T}{T}} \alpha ;-\frac{1-T}{T}\right) .
$$

Thus our setup delivers directly the value of the signal quasidistribution function at the phase space point dependent on the amplitude and the phase of the probe coherent state. Since both these parameters can be controlled experimentally without difficulties, we may simply scan the phase space by changing the amplitude and the phase of the probe field and thus determine the complete quasidistribution function. Eq. (6) shows that its ordering depends on the beamsplitter transmissivity and for $T$ near one approaches zero, which means that the scanned quasidistribution is close to the Wigner function of the signal field.

We shall now generalize Eq. (6) for arbitrary state of the probe mode by considering the disentanglement of the $S$ and $P$ modes. In order to achieve this we use the following Gaussian integration of normally ordered operators of the $S$ and $P$ modes:

$$
\begin{aligned}
& \hat{U}_{\text {out }}(0 ; 0) \\
& =\frac{4}{\pi^{2}} \int \mathrm{d}^{2} \beta: \exp \left(-2\left(\sqrt{T} \beta^{*}-\hat{a}_{P}^{\dagger}\right)\left(\sqrt{T} \beta-\hat{a}_{P}\right)\right): \\
& \quad \times: \exp \left(-2\left(\sqrt{1-T} \beta^{*}-\hat{a}_{S}^{\dagger}\right)\left(\sqrt{1-T} \beta-\hat{a}_{S}\right)\right): .
\end{aligned}
$$

Under the assumption that the $S$ and $P$ modes are uncorrelated this disentanglement yields the following expression for the quantity detected by our setup:

$$
\begin{aligned}
W_{\text {out }}(0) & =\int \mathrm{d}^{2} \beta W_{S}(\sqrt{1-T} \beta) W_{P}(\sqrt{T} \beta) \\
& =\frac{1}{1-T} \int \mathrm{d}^{2} \beta W_{S}(\beta) W_{P}(\sqrt{T /(1-T)} \beta) .
\end{aligned}
$$

This formula establishes the connection between the photon statistics of the outgoing mode and the Wigner functions of the $S$ and $P$ modes. It reflects the fundamental advantages of our setup in the direct probing of the phase space of the light field. In the case when the beamsplitter splits the light equally, we have $\sqrt{T /(1-T)}=1$ and $W_{\text {out }}(0)$ is simply a doubled overlap of the signal and probe Wigner functions. In the general case the phase space parameterization of the probe Wigner function is rescaled by the factor $\sqrt{T /(1-T)}$ which can take an arbitrary positive value depending on the beamsplitter transmissivity. This rescaling causes an effective decrease of the probe width by the factor $\sqrt{(1-T) / T}$ in all the quadratures simultaneously. This contrasts with the unbalanced double homodyne detection scheme [7, 11], where the resolution of the phase space probing along one quadrature can be improved only at the cost of deteriorating the resolution along the perpendicular direction. For the coherent probe field $|\alpha\rangle$ the $P$ mode Wigner function is of the form

$$
W_{P}(\beta)=\frac{2}{\pi} \exp \left(-2|\beta-\alpha|^{2}\right) .
$$

An easy computation shows that in this case Eq. (8) indeed reduces to Eq. (6). The rescaling of the probe Wigner function in the convolution (8) has another consequence. When $T$ tends to one, the factor multiplying the probe amplitude $\alpha$ becomes very small and to scan the interesting region of the signal phase space one has to use a probe field of large intensity.

One of the advantages of balanced homodyne detection used in quantum tomography experiments is the cancellation of the excess noise of the reference field. In our 
scheme this noise deteriorates the resolution with which the signal phase space can be probed. This can become important in the limit $T \rightarrow 1$, where strong probe fields have to be used. The influence of the excess noise can be simply estimated assuming a Gaussian thermal noise described by the following Wigner function

$$
W_{P}(\beta)=\frac{2}{\pi(2 \bar{n}+1)} \exp \left(-\frac{2}{2 \bar{n}+1}|\beta-\alpha|^{2}\right),
$$

where $\bar{n}$ is the mean number of thermal photons in the beam. An easy calculation shows that for such a noisy probe field

$$
W_{\text {out }}(0)=\frac{1}{T} W_{S}\left(\sqrt{\frac{1-T}{T}} \alpha ;-(2 \bar{n}+1) \frac{1-T}{T}\right) .
$$

In particular, when $\bar{n} \gg 1$ grows linearly with the probe intensity $|\alpha|^{2}$, the excess noise imposes a restriction on the highest ordering of the signal quasidistribution function measured at a given point.

The proposed setup is an optical realization of a model scheme of quantum measurement [12,13, where in addition to the system a filter device - a "quantum ruler" is introduced and the measured phase space probability distribution is the convolution of the system and filter Wigner functions. Our scheme is more general, since the Wigner function of the filter can be rescaled by an arbitrary factor. Consequently the rescaled probe Wigner function does not have to obey the Heisenberg uncertainty principle and may even approach the shape of a delta function, which leads to the direct measurement of the Wigner function. In contrast to quantum tomography no sophisticated computer processing of the experimental data is necessary. The quantity measured in the experiment is proportional to the quasiprobability distribution at the phase space point depending only on the amplitude and phase of the probe state.

In the remaining discussion we will introduce two generalizations. First we will make our considerations more realistic by taking into account the imperfectness of the photodetector. When the detector efficiency is $\eta$, the probability of counting $n$ photons is given by the expectation value of $: \exp \left(-\eta \hat{a}_{\text {out }}^{\dagger} \hat{a}_{\text {out }}\right)\left(\eta \hat{a}_{\text {out }}^{\dagger} \hat{a}_{\text {out }}\right)^{n} / n !:$. The second extension is the substitution of the factor $(-1)^{n}$ in Eq. (3) by $-(s+1)^{n} /(s-1)^{n+1}$, where $s$ is a real parameter. The origin and role of the parameters $\eta$ and $s$ is different: $\eta$ describes experimental limitations, while $s$ is an artificial number introduced in the numerical processing of the measured data. With these two parameters we obtain the following simple generalization of the formula (5), when expressed in terms of the $S$ and $P$ modes

$$
\begin{aligned}
& \hat{U}_{\text {out }}^{(\eta)}(0, s) \\
& =\frac{2}{\pi(1-s)} \sum_{n=0}^{\infty}\left(\frac{s+1}{s-1}\right)^{n}: e^{-\eta \hat{a}_{\text {out }}^{\dagger} \hat{a}_{\text {out }}} \frac{\left(\eta \hat{a}_{\text {out }}^{\dagger} \hat{a}_{\text {out }}\right)^{n}}{n !}:
\end{aligned}
$$

$$
\begin{aligned}
& =\frac{2}{\pi(1-s)}: \exp \left(-\frac{2 \eta}{1-s} \hat{a}_{\text {out }}^{\dagger} \hat{a}_{\text {out }}\right): \\
& =\frac{2}{\pi(1-s)}: \exp \left(-\frac{2 \eta T}{1-s}\left(\hat{a}_{S}^{\dagger}-\sqrt{(1-T) / T} \hat{a}_{P}^{\dagger}\right)\right. \\
& \left.\quad \times\left(\hat{a}_{S}-\sqrt{(1-T) / T} \hat{a}_{P}\right)\right):
\end{aligned}
$$

The third line of this equation suggests that the parameter $s$ can be used to compensate the imperfectness of the photodetector. Indeed if we selected $s=1-\eta$, we would determine the expectation value of $: \exp \left(-2 \hat{a}_{\text {out }}^{\dagger} \hat{a}_{\text {out }}\right)$ : regardless of the detector efficiency. But in this case the factor multiplying the probability of counting $n$ photons is $(1-2 / \eta)^{n}$ and its magnitude diverges to infinity with $n \rightarrow \infty$. This is not important from a theoretical point of view, since we have shown that the series converges to the expectation value of a well behaved operator. However it becomes crucial in the processing of the measured probability distribution, which is influenced by experimental errors and statistical fluctuations, and hence need not tend to zero sufficiently quickly to assure the convergence of the complete series. This reasoning might be opposed since the experimental sample of the photodetector counts is finite and thus the counts distribution is zero above certain photon number. Nevertheless the problem still exists, since the increasing factor in the generalization of the sum (3) causes that an important contribution comes from the "tail" of the experimental counts distribution, which has usually has a very poor statistics, and consequently the final result has a huge statistical error [14. The simplest way to avoid all these problems is to assume the factors multiplying the counts statistics to be bounded, which is equivalent to the condition $s \leq 0$.

An easy computation shows that if the coherent state (9) is employed as a probe, the expectation value of the generalized operator $\hat{U}_{\text {out }}^{(\eta)}(0, s)$ is again given by the quasidistribution function of the signal mode:

$$
\left\langle\hat{U}_{\text {out }}^{(\eta)}(0, s)\right\rangle=\frac{1}{\eta T} W_{S}\left(\sqrt{\frac{1-T}{T}} \alpha ;-\frac{1-s-\eta T}{\eta T}\right) .
$$

Let us now analyze the ordering of this function. It has been pointed out by Leonhardt and Paul [15] that although from a theoretical point of view an arbitrarily ordered distribution contains the complete characterization of the quantum state, experimental errors make it difficult to compute higher ordered distributions from the measured one. Thus what is interesting is the highest ordering achievable in our scheme. Analysis of the role of the parameter $s$ is the simplest, since the greater its value, the higher is the ordering obtained. Because it is restricted in its range to nonpositive values, it should be consequently set to zero. Thus we are left with two parameters: $\eta$ and $T$. It is easy to check that for fixed $\eta$ the highest ordering is still achieved when $T \rightarrow 1$, but its limit value is now $-(1-\eta) / \eta$. Under the assumption that 
$\eta$ and $T$ are close to one, the ordering of the measured distribution is effectively equal to this limiting value if the difference $1-T$ is much smaller than $1-\eta$. For currently used photodetectors, this condition can be realized experimentally. Thus the highest ordering achievable in our scheme is effectively determined by the photodetector efficiency and is equal to $-(1-\eta) / \eta$. It is noteworthy that this is exactly equal to the ordering of the distribution reconstructed tomographically from data measured in the homodyne detection with imperfect detectors [16].

The measurement of the quasiprobability distribution does not exhaust possible applications of the proposed setup. Since the probe field may be in arbitrary state, the variety of information on the quantum state which can be retrieved using this scheme is potentially very large. Another interesting extension of the presented work is its generalization to the multimode case.

We wish to acknowledge useful discussions with J. Mostowski and Cz. Radzewicz. We thank P. L. Knight for numerous comments about the final version of the manuscript. This work has been partially supported by the Polish KBN grant.

Note added. After this Letter was submitted, Ref. 117. was brought to the authors' attention. It discusses an analogous measurement scheme with a coherent state used as a probe.

* Also at the Center of Advanced Studies and Department of Physics, University of New Mexico, Albuquerque NM 87131, USA.

[1] E. P. Wigner, Phys. Rev. 40, 749 (1932). For a review see M. Hillery, R. F. O'Connell, M. O. Scully, and E. P. Wigner, Phys. Rep. 106, 121 (1984).

[2] See for example W. Vogel, D.-G. Welsch, Lectures on Quantum Optics (Akademie Verlag, Berlin, 1994).

[3] K. Vogel and H. Risken, Phys. Rev. A 40, R2847 (1989).

[4] D. T. Smithey, M. Beck, M. G. Raymer, and A. Faridani, Phys. Rev. Lett. 70, 1244 (1993); D. T. Smithey, M. Beck, J. Cooper, M. G. Raymer, and A. Faridani, Phys. Scr. T48, 35 (1993); M. G. Raymer, J. Cooper, H. J. Carmichael, M. Beck and D. T. Smithey, J. Opt. Soc. Am. B12, 1801 (1995).

[5] J. H. Shapiro and S. S. Wagner, IEEE J. Quantum Electron. QE-20, 803 (1984).

[6] M. Freyberger and W. Schleich, Phys. Rev. A 47, R30 (1993); U. Leonhardt and H. Paul, Phys. Rev. A 47, R2460 (1993).

[7] N. G. Walker, J. Mod. Opt. 34, 15 (1987).

[8] The double homodyne detection has been realized experimentally in the context of phase measurement by N. G. Walker and J. E. Caroll, Opt. Quant. Electron. 18, 355 (1986); J. W. Noh, A. Fougères, and L. Mandel, Phys. Rev. Lett. 67, 1426 (1991); Phys. Rev. A 45, 424 (1992);
46, 2840 (1992)

[9] K. E. Cahill and R. J. Glauber, Phys. Rev. 177, 1882 (1969).

[10] A. Royer, Phys. Rev. Lett. 55, 2745 (1985); Phys. Rev. A 15, 449 (1977).

[11] U. Leonhardt, Phys. Rev. A 48, 3265 (1993).

[12] K. Wódkiewicz, Phys. Rev. Lett. 52, 1064 (1984); Phys. Lett. A115, 304 (1986); A124, 207 (1987). For a review see S. Stenholm, Ann. Phys. (N.Y.) 218, 233 (1992).

[13] V. Bužek, C. H. Keitel, and P. L. Knight, Phys. Rev. A 51, 2575 (1995); 51, 2594 (1995).

[14] It has been recently noted (T. Kiss, U. Herzog, and U. Leonhardt, Phys. Rev. A 52, 2433 (1995)) that the true photon statistics can be recovered from data measured by an imperfect detector, provided that its efficiency is greater than $50 \%$. However, application of this method in calculating $W_{\text {out }}(0)$ turns out to be equivalent to setting $s=1-\eta$ in Eq. (12), which leads to problems discussed in the text.

[15] U. Leonhardt and H. Paul, Phys. Rev. Lett. 72, 4086 (1994); J. Mod. Opt. 41, 1427 (1994).

[16] U. Leonhardt and H. Paul, Phys. Rev. A 48, 4598 (1993).

[17] S. Wallentowitz and W. Vogel, Phys. Rev. A, in press.

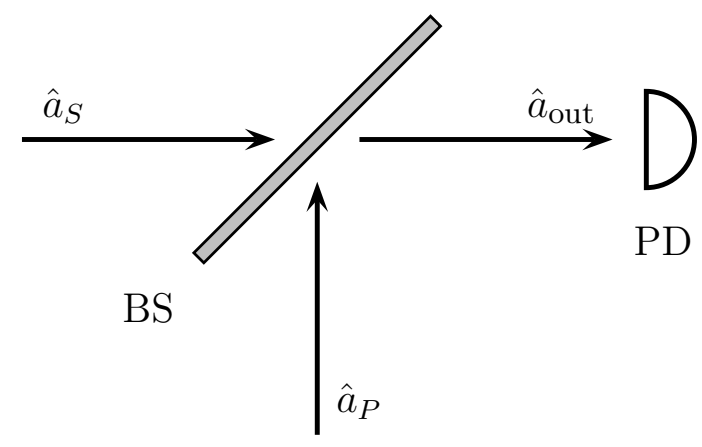

FIG. 1. Experimental setup discussed in the Letter. BS denotes the beamsplitter, $\mathrm{PD}$ is the photodetector, and the annihilation operators of the modes are indicated. 